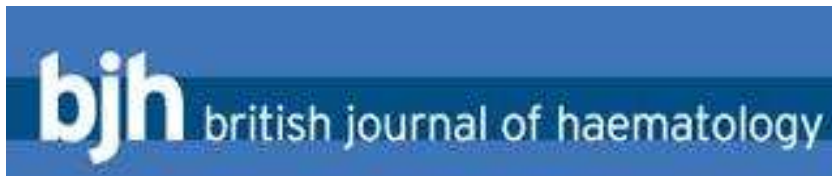

\title{
Revaccination of children after completion of standard chemotherapy for acute lymphoblastic leukaemia: a pilot study comparing different schedules
}

\begin{tabular}{|r|l|}
\hline Journal: & British Journal of Haematology \\
\hline Manuscript ID: & BJH-2010-01587 \\
\hline Manuscript Type: & Short Reports \\
\hline Author: & 11 -Oct-2010 \\
\hline Complete List of Authors: & $\begin{array}{l}\text { Lehrnbecher, Thomas; Children's Hospital III, Department of } \\
\text { Paediatric Haematology and Oncology } \\
\text { Schubert, Ralf; Children's Hospital I, Paediatric Pulmonology, } \\
\text { Allergology, and Cystic Fibrosis } \\
\text { Allwinn, Regina; University of Frankfurt, Institute of Medical } \\
\text { Virology } \\
\text { Dogan, Kader; Children's Hospital III, Pediatric Haematology and } \\
\text { Oncology } \\
\text { Koehl, Ulrike; Children's Hospital III, Pediatric Haematology and } \\
\text { Oncology } \\
\text { Grüttner, Hans Peter; University of Frankfurt, Children's Hospital }\end{array}$ \\
\hline \hline Key Words: & \begin{tabular}{l} 
Child, ACUTE LEUKAEMIA, VACCINES \\
\hline \hline
\end{tabular} \\
\hline
\end{tabular}

\section{SCHOLARONE




\title{
Revaccination of Children after Completion of Standard Chemotherapy for Acute Lymphoblastic Leukaemia: a Pilot Study Comparing Different Schedules
}

\author{
Thomas Lehrnbecher ${ }^{1}$, Ralf Schubert ${ }^{2}$, Regina Allwinn ${ }^{3}$, Kader Dogan ${ }^{1}$, Ulrike Koehl ${ }^{1}$, \\ and Hans-Peter Grüttner ${ }^{1}$ \\ ${ }^{1}$ Paediatric Haematology and Oncology, ${ }^{2}$ Paediatric Pulmonology, Allergology, and \\ Cystic Fibrosis, and ${ }^{3}$ Institute of Medical Virology, University of Frankfurt, Germany
}

Running title: Revaccination in childhood leukaemia

Key words: Child - acute lymphoblastic leukaemia - chemotherapy - revaccination tetanus - diphtheria - poliomyelitis - Haemophilus influenzae type b

To whom the correspondence should be addressed:

Thomas Lehrnbecher, MD

Paediatric Haematology and Oncology

Johann Wolfgang Goethe-University

Theodor-Stern-Kai 7

D-60590 Frankfurt

Phone: $+49-69-6301-83481$

FAX: $+49-69-6301-6700$

e-mail: thomas.lehrnbecher@kgu.de 


\begin{abstract}
Since a significant proportion of children with acute lymphoblastic leukaemia (ALL) lose immune protection to tetanus, diphtheria, and poliomyelitis, revaccination is indicated after chemotherapy. Our randomised pilot study comparing different revaccination schedules suggests that children with ALL might be revaccinated with non-live vaccines as early as 3 months after chemotherapy.
\end{abstract}

\title{
Introduction
}

Survival rates for children suffering from acute lymphoblastic leukaemia (ALL), the most common form of childhood malignancy, approach $80 \%$, and therefore, long-term effects of therapy are of increasing importance. In this respect, various studies have demonstrated that chemotherapy may cause a loss of protective antibody levels to vaccine-preventable diseases in a significant proportion of children (van Tilburg, et al 2006). Unfortunately, data on responses to revaccination after chemotherapy with nonlive vaccines such as vaccines against tetanus or diphtheria are inconsistent (van Tilburg, et al 2006). It is therefore not surprising that considerable discrepancies exist between revaccination schedules, which are primarily based on theoretical considerations and expert opinion. Whereas early timing of revaccination (e.g., at 3 months after chemotherapy) may minimise the time leaving the vulnerable patient unprotected, others recommend revaccination not before 6 to 12 months after completion of chemotherapy to increase the immunogenicity of vaccination (Patel, et al 2007, Zignol, et al 2004). We performed a randomised pilot study to compare the efficacy of vaccines against tetanus, 
diphtheria, poliomylitis and Haemophilus influenzae type b (Hib) in children revaccinated at 3, 6 or 9 months after completion of non-high-risk ALL chemotherapy, respectively.

\section{Patients and Methods}

The study was conducted from January 2001 through December 2003 in the Paediatric Haematology and Oncology, University of Frankfurt, Germany. Eligibility criteria included completed chemotherapy for de novo, non-high-risk ALL, no evidence of immunodeficiency before diagnosis of malignancy, and vaccination against diphtheria, tetanus, and poliovirus prior to diagnosis of ALL. The study was approved by the local Ethical Committee. Written informed consent was obtained from the patient and/or parents before patients were randomly assigned to one of the groups using a centralised computer system. According to randomisation, patients received booster DTaP-IPV and Hib conjugate vaccines [Infanrix ${ }^{\mathrm{TM}}$-IPV+Hib (GlaxoSmithKline)] either at 3, 6 or 9 months after completion of chemotherapy; a hepatitis B vaccine [Gen H-B-Vax ${ }^{\mathrm{TM}}$ (Pasteur MSD/Chiron Behring)] was simultaneously given to some of the patients. Blood samples were obtained at the time of completion of chemotherapy, on the day of revaccination and 3 months after revaccination to assess lymphocyte subsets, immunoglobulin subclasses and serum antibody concentrations or titres. Lymphocyte subsets and immunoglobulin subclasses were analysed as previously reported (Huenecke, et al 2008, Lehrnbecher, et al 2009). Antibody concentrations specific to tetanus, diphtheria, and Hib were measured by standardised enzyme-linked immunosorbent assays (ELISA) following the manufacturer's instruction (PROGEN Biotechnik, Heidelberg, Germany). A neutralisation assay was used to detect neutralising antibodies against poliovirus 
serotypes 1-3 (Rabenau and Weber 1994). Titres against poliovirus $\geq 1: 8$ were considered to correlate with protection; antitetanus and antidiphtheria antibody concentrations of $\geq 0.01$ and $\geq 0.1 \mathrm{IU} / \mathrm{mL}$, and Hib antibody concentrations $\geq 0.15$ and $\geq 1.0 \mu \mathrm{g} / \mathrm{mL}$ were correlated with short-term and long-term protection, respectively (Patel, et al 2007).

\section{Statistical analysis}

Lymphocyte subsets and immunoglobulin subclasses were compared to age-matched reference values (Lehrnbecher, et al 2009). For statistical analysis, antibody concentrations or titres that were below the limit of the assay's sensitivity were assigned values equal to one-half of the lower detection limit. All antibody concentrations or titres were log-transformed to calculate geometric mean concentration or geometric mean, with 95\% CI. Antibody levels before and after revaccinations were compared using Wilcoxon's test. Groups were compared using a multi-dimensional H-test (KruskalWallis). A $P$ value $<0.05$ (2-sided) was considered significant.

\section{Results}

A total of 24 patients [14 boys, 10 girls; median age 7.3 years (range, 4.1-20.7)] were included in the study. All patients were treated according to the clinical trials BFM-ALL $95(n=15)$ or BFM-ALL $2000(n=9)$. Seven patients received revaccination for tetanus, diphtheria, poliomyelitis, and Hib at 3 months after completion of chemotherapy, and 8 and 9 patients at 6 and 9 months, respectively. 
Lymphocyte subsets and immunoglobulin subclasses. When analysing the lymphocyte subsets at the time of vaccination, the median absolute number of $\mathrm{CD}^{+} \mathrm{CD}^{+}$cells was 766, 752 and 662 per $\mu 1$ for patients at 3, 6, and 9 months after completion of chemotherapy, respectively [range, 175-1230, 450-1574, and 326-1319, respectively; differences not significant (ns)]; similarly, the median number of $\mathrm{CD}^{+} \mathrm{CD} 3^{+}$cells was 541 (range, 85-1690), 628 (352-1575), and 558 (275-914) and for CD19+ cells 339 (841179), 526 (358-2336), and 413 (245-775) per $\mu 1$ for these patient groups (differences ns). In other words, at the time of vaccination, only one of 7 patients vaccinated at 3 months after completion of chemotherapy was below the age-matched $90 \%$ reference interval for $\mathrm{CD}^{+} \mathrm{CD}^{+}$and $\mathrm{CD} 19^{+}$cells, respectively, whereas this was seen for $\mathrm{CD}^{+} \mathrm{CD}^{+}$cells for one patient of each group. Similarly, there was no significant difference of the levels of immunoglobulin subclasses and the percentages of patients below the age-matched reference values assessed at the time of vaccination between the patients revaccinated at 3, 6, and 9 months after completion of chemotherapy, respectively (data not shown).

Tetanus and Diphtheria. At the time of completion of chemotherapy as well as at the time of revaccination, about two thirds of the patients evenly distributed in the different patient groups (e.g., revaccination at 3,6, and 9 months after chemotherapy, respectively) had tetanus and diphtheria antibody concentrations $<0.1 \mathrm{IU} / \mathrm{mL}$, indicating that these patients required booster vaccination (Table). For both tetanus and diphtheria antibody concentrations, there was a significant increase after vaccination (Table). Importantly, all patients achieved protecting antibody concentration $\geq 0.1 \mathrm{IU} / \mathrm{mL}$ for tetanus and 
diphtheria, whether vaccinated after 3,6 or 9 months after completion of chemotherapy, respectively.

Poliovirus. Two third of the patients $(n=12)$ evenly distributed in the different patient groups did not have protective levels of antibody titre to all 3 poliovirus serotypes at the time of completion of chemotherapy and at the time of revaccination, respectively (Table). Specifically, at the time of revaccination, protective titres to serotypes 1,2 , and 3 were present in $81 \%, 81 \%$ and $48 \%$ of patients, respectively. For all groups, there was a significant increase of antibody titres after vaccination, and importantly, all 24 patients achieved protection to all serotypes (Table). In the three patients reassessed after 3 years of vaccination (one of each group revaccinated after 3, 6, and 9 after the end of chemotherapy, respectively), antibody titres remained unchanged.

Hib. All but three patients had received at least one dose of Hib conjugate vaccine prior to diagnosis of leukaemia. In contrast to tetanus, diphtheria, and polio, more than half of the patients demonstrated long-term immunity with antibody concentrations $\geq 1 \mu \mathrm{g} / \mathrm{mL}$ at the time of completion of therapy $(11 / 20 ; 55 \%)$ and at the time of revaccination $(14 / 21$; $67 \%$ ), respectively (Table). After revaccination, the antibody concentration significantly increased, and all patients achieved antibody concentration $\geq 1 \mu \mathrm{g} / \mathrm{mL}$ indicating longterm protection (Table). Notably, in $21 / 24$ patients (87\%), the antibody concentration rose to levels $>5 \mu \mathrm{g} / \mathrm{mL}$ which correlates with protection against Hib colonization.

Adverse events. Only one patient revaccinated at 9 months after completion of therapy developed fever after vaccination. No other local or systemic adverse events were noted.

\section{Discussion}


The data of this pilot trial corroborate previous reports that a significant proportion of children undergoing therapy for non-high-risk ALL lose protection to tetanus, diphtheria, polio, and Hib (van Tilburg, et al 2006). Our data suggest that revaccination results in protective, long-lasting antibody concentration, independently whether revaccination is given 3, 6 or 9 months after completion of therapy, respectively. In line with the results of a previous study, in the majority of patients, lymphocyte subsets and immunoglobulin levels were already 3 months after the end of chemotherapy above the lower limit of the age-matched 90\% reference intervals (Lehrnbecher, et al 2009). Notably, non-high-risk patients exhibit a faster immune recovery than high-risk patients (Ek, et al 2005, Lehrnbecher, et al 2009). A recent review demonstrated that protection rates after completion of chemotherapy vary widely depending on underlying malignancy and chemotherapeutic regimen (e.g., 20-98\% for tetanus, $17-98 \%$ for diphtheria, $62-100 \%$ for poliomyelitis, and 35-100\% for Hib) (van Tilburg, et al 2006), and most experts agree that revaccination in these children is indicated. Although in most studies, a significant increase of the levels of protective antibodies was seen after revaccination for tetanus, diphtheria, and poliomyelitis, even when administered already during maintenance chemotherapy for ALL, a comparison of these studies is difficult due to differences in the intensity of chemotherapy and study design (Calaminus, et al 2007, Ercan, et al 2005, Patel, et al 2007, Zengin and Sarper 2009, Zignol, et al 2004). Unfortunately, no study to date compared the efficacy of different revaccination schedules after chemotherapy, which is always a compromise between reduced immunogenicity after immunosuppression and the risk to leave a patient unprotected for a period of time. Our data suggest that children with non-high-risk ALL might be revaccinated with vaccines to 
tetanus, diphtheria, and poliomyelitis as early as 3 months after completion of chemotherapy, since efficacy seems to be similar for the different schedules assessed (e.g., at 3, 6, or 9 months after chemotherapy). In contrast to tetanus, diphtheria, and poliomyelitis, the majority of patients demonstrated long-term protective antibody concentrations to $\mathrm{Hib} \geq 1 \mu \mathrm{g} / \mathrm{mL}$ already at the time of completion of chemotherapy. However, revaccination increased antibody concentrations to $\geq 5 \mu \mathrm{g} / \mathrm{mL}$ in most of the patients, which correlates with protection against Hib colonization and therefore suggests a benefit of revaccination (Fernandez, et al 2000). Although we recognise the small study population, we believe that the unequivocal results of this trial may help to minimise the time of children being unprotected against vaccine-preventable diseases after chemotherapy for non-high-risk ALL, and this approach might be transferred to patients receiving less intensive chemotherapy, for example to patients with solid tumours. 
Table. Geometric mean concentrations (GMC) and titres (GMT) of antibodies to tetanus, diphtheria, poliomyelitis, and Haemophilus influenzae type b (Hib) in children vaccinated at 3 months (group I, n=7), 6 months (group II, n=8), or 9 months (group III, n=9) after completion of chemotherapy for non-high-risk acute lymphoblastic leukaemia. Antibody titres were assessed at the time of completion of therapy ("after therapy"), on the day of revaccination (“pre-vaccination”), and 3 months after revaccination (“post-vaccination”). $\%$, percentage of protected patients

$* P<0.05$ for prior to versus after revaccination

** $P<0.005$ for prior to versus after revaccination

*** $P<0.001$ for prior to versus after revaccination

\# $P<0.01$ for comparison between group 3 and groups 1 and 2 , respectively 


\section{References}

Calaminus, G., Hense, B., Laws, H.J., Groeger, M., MacKenzie, C.R. \& Gobel, U. (2007) Diphtheria (D) and tetanus (T) antibody values in children with acute lymphoblastic leukaemia (ALL) after treatment according to Co-ALL 05/92. Klin Padiatr, 219, 355-360.

Ek, T., Mellander, L., Andersson, B. \& Abrahamsson, J. (2005) Immune reconstitution after childhood acute lymphoblastic leukemia is most severely affected in the high risk group. Pediatr Blood Cancer, 44, 461-468.

Ercan, T.E., Soycan, L.Y., Apak, H., Celkan, T., Ozkan, A., Akdenizli, E., Kasapcopur, O. \& Yildiz, I. (2005) Antibody titers and immune response to diphtheria-tetanuspertussis and measles-mumps-rubella vaccination in children treated for acute lymphoblastic leukemia. Journal of Pediatric Hematology and Oncology, 27, 273-277.

Fernandez, J., Levine, O.S., Sanchez, J., Balter, S., LaClaire, L., Feris, J. \& RomeroSteiner, S. (2000) Prevention of Haemophilus influenzae type b colonization by vaccination: correlation with serum anti-capsular IgG concentration. Journal of Infectious Diseases, 182, 1553-1556.

Huenecke, S., Behl, M., Fadler, C., Zimmermann, S.Y., Bochennek, K., Tramsen, L., Esser, R., Klarmann, D., Kamper, M., Sattler, A., von Laer, D., Klingebiel, T., Lehrnbecher, T. \& Koehl, U. (2008) Age-matched lymphocyte subpopulation reference values in childhood and adolescence: application of exponential regression analysis. European Journal of Haematology, 80, 532-539. 
Lehrnbecher, T., Schubert, R., Behl, M., Koenig, M., Rose, M.A., Koehl, U., Meisel, R. \& Laws, H.J. (2009) Impaired pneumococcal immunity in children after treatment for acute lymphoblastic leukaemia. British Journal of Haematology, 147, 700705.

Patel, S.R., Ortin, M., Cohen, B.J., Borrow, R., Irving, D., Sheldon, J. \& Heath, P.T. (2007) Revaccination of children after completion of standard chemotherapy for acute leukemia. Clinical Infectious Diseases, 44, 635-642.

Rabenau, H. \& Weber, B. (1994) Evaluation of a new automated microneutralization assay for the quantitative detection of neutralizing antibodies against enteroviruses. Zentralbl Bakteriol, 280, 534-539.

van Tilburg, C.M., Sanders, E.A., Rovers, M.M., Wolfs, T.F. \& Bierings, M.B. (2006) Loss of antibodies and response to (re-)vaccination in children after treatment for acute lymphocytic leukemia: a systematic review. Leukemia, 20, 1717-1722.

Zengin, E. \& Sarper, N. (2009) Humoral immunity to diphtheria, tetanus, measles, and hemophilus influenzae type $\mathrm{b}$ in children with acute lymphoblastic leukemia and response to re-vaccination. Pediatr Blood Cancer, 53, 967-972.

Zignol, M., Peracchi, M., Tridello, G., Pillon, M., Fregonese, F., D'Elia, R., Zanesco, L. \& Cesaro, S. (2004) Assessment of humoral immunity to poliomyelitis, tetanus, hepatitis B, measles, rubella, and mumps in children after chemotherapy. Cancer, 101, 635-641. 


\begin{tabular}{|c|c|c|c|c|c|c|c|}
\hline & & \multicolumn{6}{|c|}{ Visits } \\
\hline & & \multicolumn{2}{|c|}{ After Therapy } & \multicolumn{2}{|c|}{ Pre-Vaccination } & \multicolumn{2}{|c|}{ Post-Vaccination } \\
\hline & & Titer & $\%$ & Titer & $\%$ & Titer & $\%$ \\
\hline$\frac{\partial}{\underline{\underline{\varepsilon}}}$ & Group I & $\begin{array}{c}0.13 \\
(0.03-0.51)\end{array}$ & 43 & $\begin{array}{c}0.12 \\
(0.03-0.50)\end{array}$ & 43 & $\begin{array}{c}1.61^{*} \\
(1.12-2.32)\end{array}$ & 100 \\
\hline 变 & Group II & $\begin{array}{c}0.03 \\
(0.02-0.06)\end{array}$ & 17 & $\begin{array}{c}0.05 \\
(0.02-0.12)\end{array}$ & 15 & $\begin{array}{c}1.63^{*} \\
(1.00-2.66)\end{array}$ & 100 \\
\hline$\sum_{0}^{0}$ & Group III & $\begin{array}{c}0.28 \\
(0.07-1.15)\end{array}$ & 63 & $\begin{array}{c}0.19 \\
(0.04-0.83)\end{array}$ & 50 & $\begin{array}{c}2.27^{\star} \\
(1.82-2.82)\end{array}$ & 100 \\
\hline$\frac{\partial}{\xi}$ & Group I & $\begin{array}{c}0.14 \\
(0.06-0.38)\end{array}$ & 43 & $\begin{array}{c}0.12 \\
(0.04-0.37)\end{array}$ & 43 & $\begin{array}{c}3.72^{*} \\
(1.96-7.04)\end{array}$ & 100 \\
\hline ํ. & Group II & $\begin{array}{c}0.07 \\
(0.03-0.18)\end{array}$ & 33 & $\begin{array}{c}0.06 \\
(0.04-0.07)\end{array}$ & 29 & $\begin{array}{c}2.73^{\star} \\
(1.22-6.14)\end{array}$ & 100 \\
\hline$\sum_{0}^{0}$ & Group III & $\begin{array}{c}0.11 \\
(0.03-0.40) \\
\end{array}$ & 25 & $\begin{array}{c}0.10 \\
(0.04-0.27) \\
\end{array}$ & 38 & $\begin{array}{c}2.29^{*} \\
(0.90-5.83) \\
\end{array}$ & 100 \\
\hline$\underline{\vec{\partial}}$ & Group I & $\begin{array}{c}0.52 \\
(0.15-1.83)\end{array}$ & 33 & $\begin{array}{c}0.81 \\
(0.15-4.31)\end{array}$ & 50 & $\begin{array}{c}8.05^{\star} \\
(6.04-10.7)\end{array}$ & 100 \\
\hline $\begin{array}{l}\text { 금 } \\
\text { 읃 }\end{array}$ & Group II & $\begin{array}{c}1.28 \\
(0.66-2.49)\end{array}$ & 83 & $\begin{array}{c}1.34 \\
(0.72-2.49)\end{array}$ & 71 & $\begin{array}{c}9.39^{\star} \\
(8.33-10.6)\end{array}$ & 100 \\
\hline$\sum_{0}$ & Group III & $\begin{array}{c}1.21 \\
(0.55-2.68) \\
\end{array}$ & 50 & $\begin{array}{c}1.56 \\
(0.90-2.69) \\
\end{array}$ & 75 & $\begin{array}{c}7.17^{*} \\
(5.37-9.59) \\
\end{array}$ & 100 \\
\hline$\frac{\widehat{x}}{\stackrel{x}{\dot{a}}}$ & Group I & $\begin{array}{c}2.73 \\
(0.15-50.9)\end{array}$ & 30 & $\begin{array}{c}9.23 \\
(0.80-108)\end{array}$ & 57 & $\begin{array}{c}599^{* *} \\
(438-820)\end{array}$ & 100 \\
\hline $\begin{array}{l}\text { E } \\
.0\end{array}$ & Group II & $\begin{array}{c}1.47 \\
(0.10-21.3)\end{array}$ & 20 & $\begin{array}{c}1.74 \\
(0.16-19.3)\end{array}$ & 33 & $\begin{array}{c}494^{\star * *} \\
(292-833)\end{array}$ & 100 \\
\hline$\sum_{0}^{0}$ & Group III & $\begin{array}{c}3.89 \\
(0.47-32.1)\end{array}$ & 50 & $\begin{array}{c}0.98 \\
(0.09-10.2)\end{array}$ & 25 & $\begin{array}{c}1097^{* * *}, \# \\
(921-1308)\end{array}$ & 100 \\
\hline
\end{tabular}

